\title{
CANNABIS USE IN FIRST EPISODE PSYCHOSIS PATIENTS: CLINICAL OUTCOMES
}

\section{Santos Silva, R. Araújo, M. Barbosa, J. Miranda, M. Duarte}

Centro Hospitalar de Leiria-EPE, Serviço de Psiquiatria e Saúde Mental, Leiria, Portugal luis.mds.silva@gmail.com

Introduction:

The prevalence of cannabis use is significant in the general population, even more so if we focus on the patients suffering psychosis. It is also known that cannabis can modify the course of an established schizophrenia and evidence also suggests an increased risk of developing psychotic symptoms in consumers of this substance.

The authors' objectives were to study the population of patients admitted at the subspecialty consultation of first psychotic episode (FPE) with a special focus on the additive behaviors. Possible epidemiological and clinical differences between cannabis users and non-users were explored.

\section{Material and methods:}

We analyzed the computerized clinical processes (consultations, emergency episodes, hospitalizations and complementary diagnostic tests) of all patients $(n=86)$ included in the FPE consultation from January 2015 to August 2018, most of whom were referred after discharge from psychiatry inward. Patients admitted to this consultation are adults aged 18 to 40 years, with no history of psychotic illness or prior use of antipsychotic drugs.

\section{Results}

Of the 86 patients analyzed, most were male ( $71 \%$ ) and at the time of the first psychotic episode, the most common provisional diagnosis was Unspecified Nonorganic Psychosis (F29 47\%), followed by Psychosis due to the Use of Cannabinoids (F12.5 - 36\%). ) and, in equal percentage $(5 \%)$, Psychosis due to the Use of Multiple Drugs (F19.5) and Severe Depressive Episode with Psychotic Symptoms (F32.3).

$59 \%$ of patients had urine cannabinoid positivity during the first psychotic episode.

These patients presented:

- an earlier age of onset (most $<25 \mathrm{~A}$ )

- higher readmission rates (23.5\% vs $12.5 \%)$

- shorter average length of hospitalization (18 vs 22.7 days)

- lower rates of compulsive hospitalizations (20\% vs $45 \%)$

No significant differences were found with respect to the antipsychotic formulation chosen for treatment.

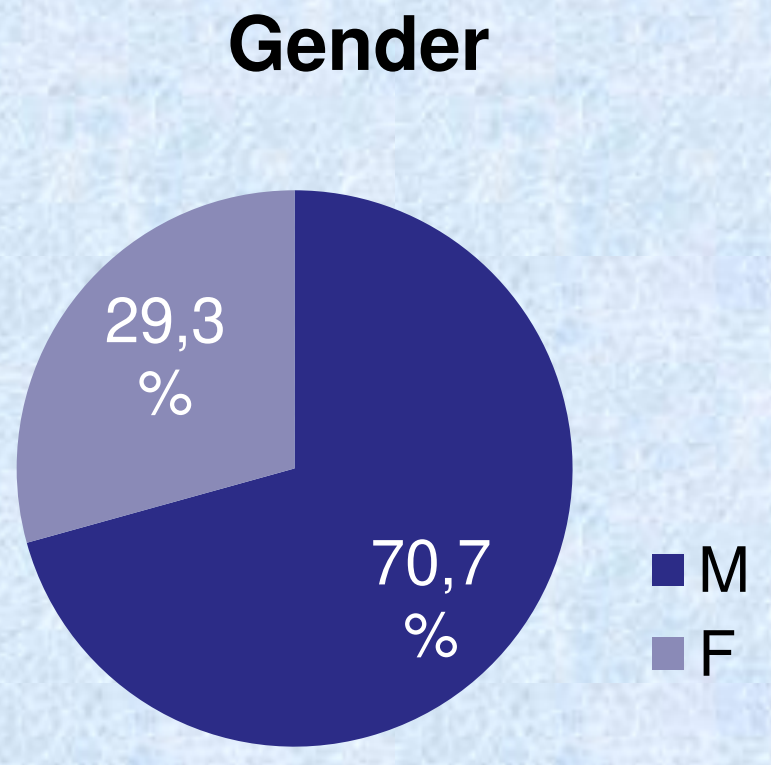

\section{Cannabis Use}

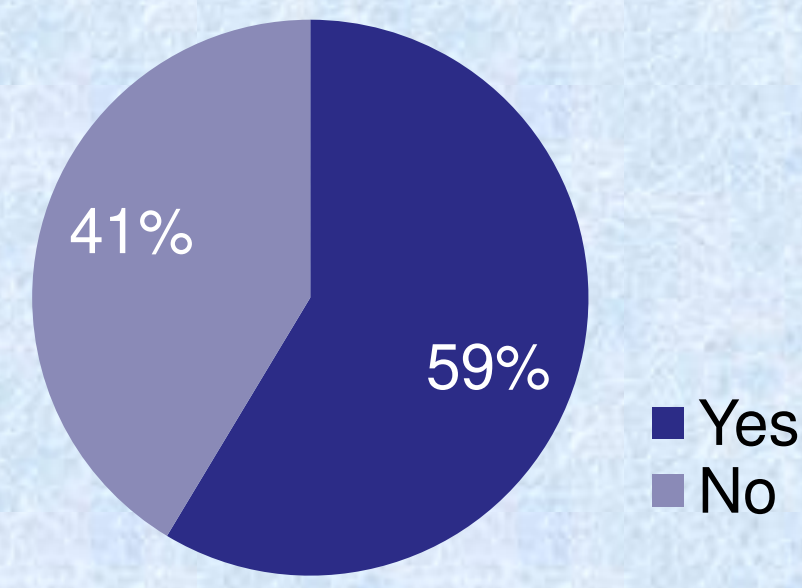

Age Range

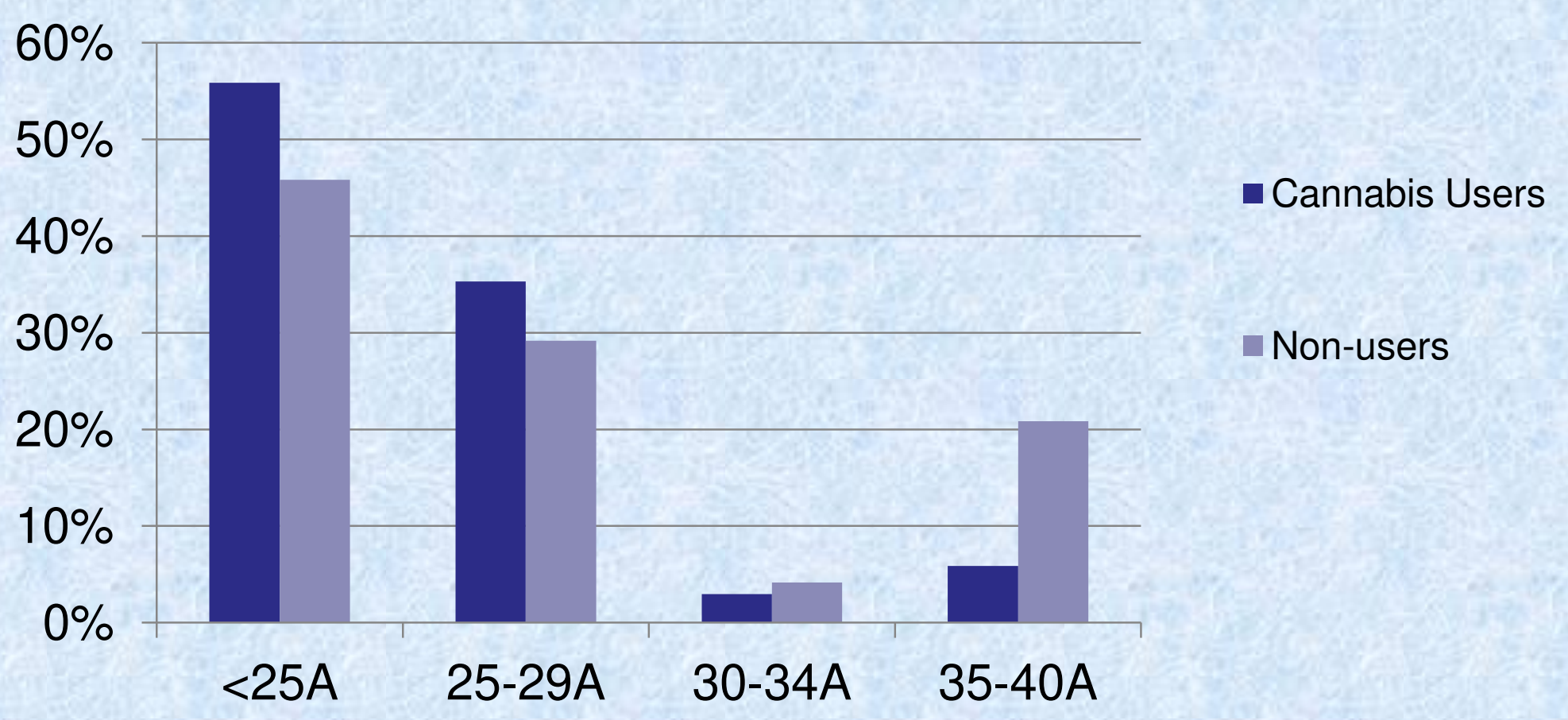

Diagnosis (ICD-10)

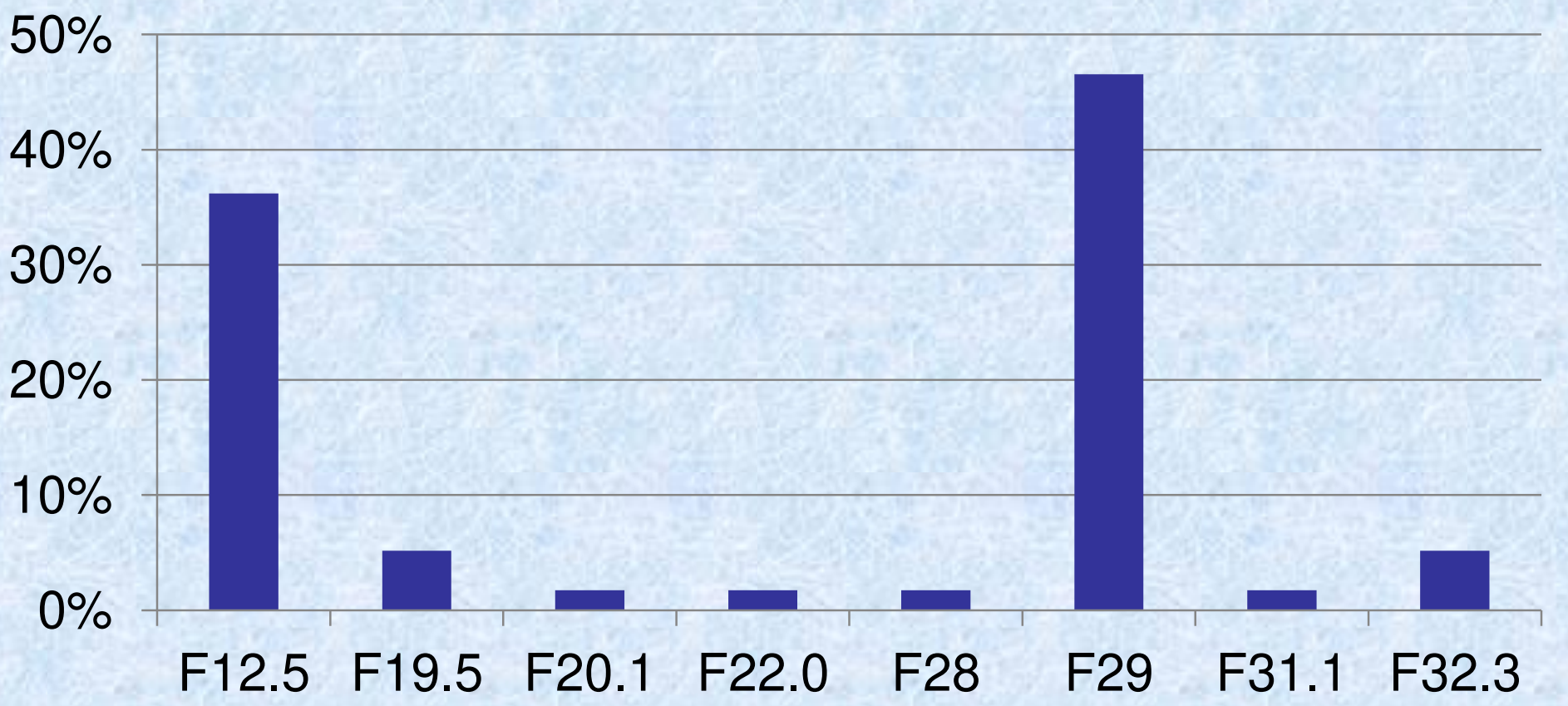

\section{Conclusions:}

The consumption of cannabis should not be neglected given its potential to trigger an early onset of psychotic symptomatology as well as serve as a maintenance factor, motivating a greater number of rehospitalizations - results in agreement with most of the existing literature on the subject. Given these results, it would be important to take preventive action to reduce the consumption of this substance in the general population and in patients with a first psychotic episode in order to improve outcomes. 